\title{
Design and Characterization of the OpenWrist: A Robotic Wrist Exoskeleton for Coordinated Hand-Wrist Rehabilitation
}

\author{
Evan Pezent ${ }^{\dagger}$, Chad G. Rose ${ }^{\dagger}$, Ashish D. Deshpande ${ }^{\ddagger}$, and Marcia K. O’Malley ${ }^{\dagger}$
}

\begin{abstract}
Robotic devices have been clinically verified for use in long duration and high intensity rehabilitation needed for motor recovery after neurological injury. Targeted and coordinated hand and wrist therapy, often overlooked in rehabilitation robotics, is required to regain the ability to perform activities of daily living. To this end, a new coupled hand-wrist exoskeleton has been designed. This paper details the design of the wrist module and several human-related considerations made to maximize its potential as a coordinated hand-wrist device. The serial wrist mechanism has been engineered to facilitate donning and doffing for impaired subjects and to insure compatibility with the hand module in virtual and assisted grasping tasks. Several other practical requirements have also been addressed, including device ergonomics, clinician-friendliness, and ambidextrous reconfigurability. The wrist module's capabilities as a rehabilitation device are quantified experimentally in terms of functional workspace and dynamic properties. Specifically, the device possesses favorable performance in terms of range of motion, torque output, friction, and closed-loop position bandwidth when compared with existing devices. The presented wrist module's performance and operational considerations support its use in a wide range of future clinical investigations.
\end{abstract}

\section{INTRODUCTION}

Robot-augmented therapy is a clinically verified path forward to improving rehabilitation outcomes for several neuromuscular conditions, such as cerebrovascular accidents (CVA or stroke) and spinal cord injuries [1]. CVAs alone impact approximately 795,000 individuals each year, and the related costs are projected to rise above the 2012 estimate of $\$ 316.6$ billion as mortality rates continue to decline [2].

Robotic rehabilitative devices enable the high intensity, long duration interventions needed for regaining motor function, and quantitative metrics for tracking therapeutic outcomes [3]. Regaining the ability to perform activities of daily living (ADL) requires targeted rehabilitation of the upper extremity, in particular, the wrist and hand. Several devices have been designed for this purpose, [4]-[11], but few allow for coordinated hand and wrist movement [12]. This separated approach overlooks the kinematic and dynamic linkings of the hand and wrist which arise from anatomy [13], as well as their position-dependent passive properties [14]-[16]. The READAPT, the coupling of a wrist exoskeleton developed in the MAHI Lab and the Maestro hand exoskeleton developed in the ReNeu Lab, was proposed to enable the coordinated

This work was supported by a training fellowship from the Gulf Coast Consortia, on the IGERT: Neuroengineering from Cells to Systems, National Science Foundation (NSF) 1250104, NSF grants NSF-CPS1135949/1135916, and NSTRF-NNX13AM70H.

${ }^{\dagger}$ Mechatronics and Haptic Interfaces Laboratory, Dept of Mechanical Engineering, Rice University, Houston, TX 77005.

${ }^{\ddagger}$ ReNeu Robotics Lab, Dept. of Mechanical Engineering, University of Texas, Austin, TX 78712.

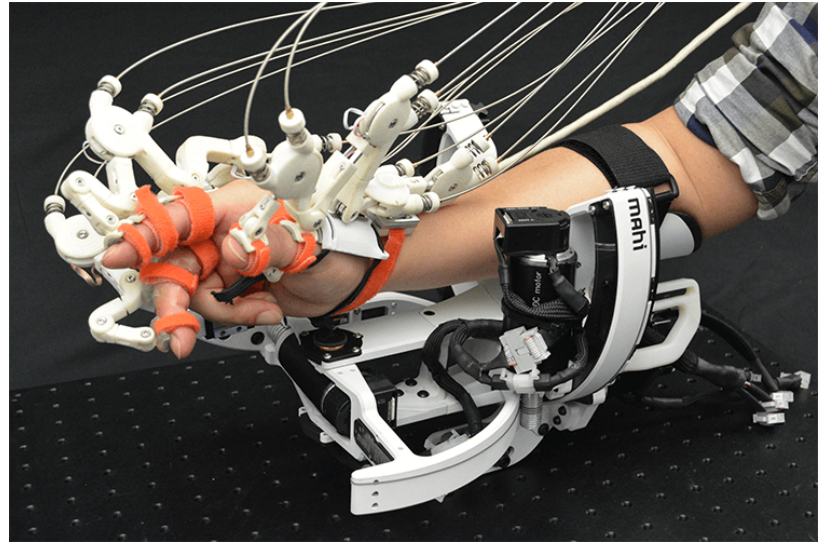

Fig. 1. MAHI OpenWrist exoskeleton module shown with the ReNeu Maestro hand exoskeleton module in the combined READAPT configuration.

hand and wrist movements required in ADL as suggested by the interconnected nature of hand-wrist musculature [17]. However, the requirements for designing coordinated handwrist exoskeletons remains relatively unknown due the sparse landscape of such devices.

\section{A. Identified Design Requirements}

A preliminary implementation of the READAPT, which utilized the existing RiceWrist-S exoskeleton [7], identified finger metacarpalphalangeal (MCP) flexion/extension range of motion (ROM) limits (subsequently addressed in [18]), wrist static friction and inertia, and undesired interactions between the hand and wrist modules as key contributors to hand-wrist discoordination in redundant MCP and wrist flexion/extension pointing tasks [17]. Additionally, pre-clinical trials with the RiceWrist-S in a standalone mode [7], as well as experience and clinician feedback from other clinical studies [19], highlighted the necessity of the user's ability to easily don/doff devices. This is especially true during studies with fragile skinned subjects where donning/doffing closeddesign exoskeletons (e.g. [7], [10], [19]) is not only difficult and time consuming, but also potentially hazardous. In order of importance, future hand-wrist exoskeletons, including the READAPT, would need to (1) provide a harmonious interface between the the hand and wrist modules, (2) enable don/doff of impaired individuals with an easily accessed open design, (3) address ergonomics and user comfort, and (4) minimize the discoordinating effects of friction and inertia. Further increasing dynamic performance over previous devices, and enabling compatibility with surface electromyography (sEMG) and passive marker motion capture were also included as design requirements specific to the READAPT. 
These goals are met by the new wrist module of the READAPT exoskeleton, the OpenWrist, shown in Fig. 1 with the Maestro hand exoskeleton and Fig. 2 in a standalone configuration. Section II presents the design and characterizes the OpenWrist's functional work space defined by range of motion and torque output. Many practical considerations are discussed in Section II-D, as well as the ergonomic considerations required for stand-alone wrist movements in Section II-E. Section III presents the characterization of the module's dynamic properties and compares them with those of the preliminary implementation. Finally, Section IV provides context for the results before concluding.

\section{DESIGN AND IMPLEMENTATION}

The OpenWrist is the evolution of the RiceWrist-S, previously presented in [7], with major refinements to each degree of freedom (DOF) to increase performance, functionality, and most importantly compatibility with the Maestro hand exoskeleton. Like its predecessor, it employs a serial RRR mechanism for manipulation of the user's wrist and forearm. The first rotational joint actuates pronation/supination (PS) of the forearm, while the second and third actuate flexion/extension (FE) and radial/ulnar deviation (RU) of the wrist, respectively. A fourth passive linear degree of freedom between the third joint and the point of human interface (i.e. the Maestro hand exoskeleton or the optional hand grip discussed in Section II-E) allows for small misalignments between the user's and robot's joints. Each actuated DOF is powered by a brushed DC motor. To ensure backlash free operation, power is transmitted through capstan-cable drives, which involves winding a high tensile strength cable around a small diameter threaded spool and terminating the cable on the ends of a larger diameter capstan arc. The novel features of each individual DOF and the entire unit are detailed in the subsections that follow, and device capabilities are provided in Table I.

\section{A. Joint 1: Pronation/Supination}

The PS joint has been designed to address a major concern for robotic exoskeletons: donning and doffing. All MAHI Lab designs thus far have required that the user insert their hand through an ring encompassing the PS joint. This task, trivial for non-impaired users, proves challenging for impaired subjects with reduced motor control and spasticity. Furthermore, a closed design requires that the Maestro be awkwardly donned after the user has inserted their arm into the wrist exoskeleton. Eliminating this shortcoming was accomplished by switching from a traditional closed radial bearing to an open curvilinear rail and slider solution (Fig. 3-a). Four $60^{\circ}, 100 \mathrm{~mm}$ radius rail sections are mounted to a central hub (Fig. 4 -a) to provide $240^{\circ}$ of rail space. To support expected moment loads, two slider mechanisms are used, each mounted to a fixed frame and elbow support section, visible in Fig. 2. Thus, it is the rails and hub that move instead of the sliders themselves. The spacing of the sliders is such that approximately $170^{\circ}$ of motion is achievable in the PS joint. The decision to have the rail

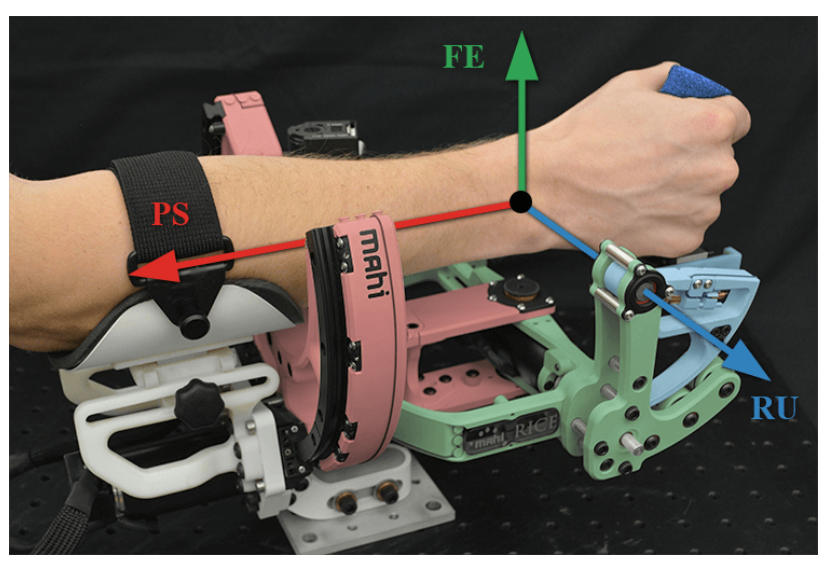

Fig. 2. OpenWrist- 3 DOF forearm and wrist exoskeleton for pathology agnostic rehabilitation in a standalone configuration. PS (red), FE (green), and RU (blue) links are highlighted to match their respective axes.

hub rotate was made so that it could simultaneously serve as a capstan arc in the transmission system. Unlike the RiceWrist-S, which used a direct drive motor, the PS joint in the OpenWrist employs a capstan-cable transmission. As a result, the new device more than doubles torque output from $1.69 \mathrm{Nm}$ to $3.50 \mathrm{Nm}$.

\section{B. Joint 2: Flexion/Extension}

With the addition of the relatively heavy rails and hub, significant changes to distal joints were necessary to offset the added inertia to the PS joint. First, the distance from the PS joint to the center of the FE axis was shortened. This change not only removed unnecessary material and weight, but also allowed for the elimination of the idler pulley mechanism present in the RiceWrist-S. It is worth noting that the FE actuator was also relocated from the dorsal side of the hand to the palmar side as shown in Fig. 3-c. Second, the RU actuator was moved approximately 2 inches closer to the PS axis by creating a gap in the FE capstan and shaft for the motor (Fig. 3-d).

\section{Joint 3: Radial/Ulnar Deviation}

Due to the placement of the RU actuator, the point of contact between the actuator shaft and capstan arc requires relocation so that an appropriate range of motion is achievable. Previously, the RiceWrist-S accomplished this via a method described in [20] which involved spanning and tensioning cable between a threaded motor shaft and a second threaded aluminum shaft. Issues with robustness and maintaining cable tension led to a modification which introduced two idler pulleys as a means to relocate the point of contact, as seen in Fig. 3. Further improvements to this idler pulley method were made with the OpenWrist. To reduce overall form-factor, three smaller pulleys were substituted for the two large pulleys. In addition, the threaded spool was doubly supported to prevent deflection in the spool as the cable is tensioned, thus reducing binding and friction.

To maximize compatibility with the Maestro hand exoskeleton, two additional key changes were made. First, the overhanging bridge coupling the RU DOF to the hand, which 

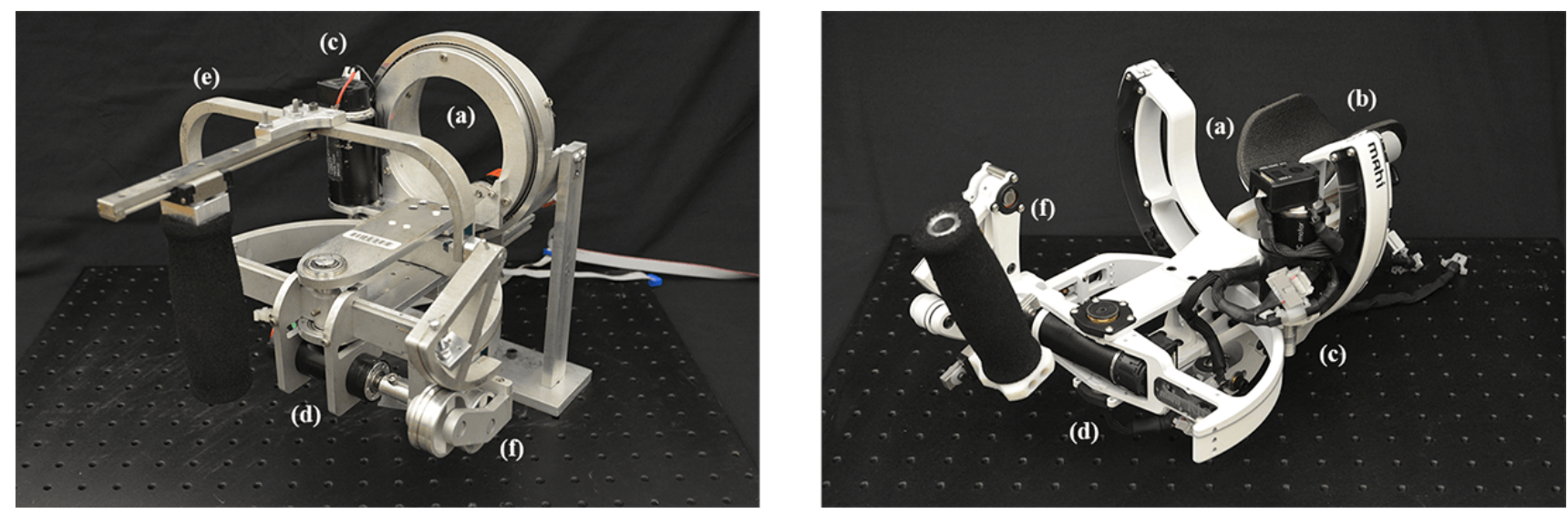

Fig. 3. RiceWrist-S (left) and OpenWrist (right) - (a) PS joint now open, (b) padded elbow support introduced, (c) FE actuator moved to palmar side, (d) RU actuator moved closer to PS axis, (e) RU bridge eliminated to minimize interference with Maestro, (f) RU module moved to dorsal side.

TABLE I

Device Capabilities Compared with Requirements for ADL and other Wrist Devices (MIT-Manus [9], IIT Wrist Robot [11], Wrist Gimbal [10], MAHI Exo-II [8], AND RiceWrist-S [7])

\begin{tabular}{lcccccccccccccc} 
& \multicolumn{1}{c}{ Range of Motion [deg] } & \multicolumn{4}{c}{ Max Continuous Torque [Nm] } \\
Joint & ADL & MIT & IIT & WG & ME-II & RW-S & OpenWrist & ADL & MIT & IIT & WG & ME-II & RW-S & OpenWrist \\
\hline PS & 150 & 140 & 160 & 180 & 180 & 180 & $\mathbf{1 7 0}(85$ P, 85 S $)$ & 0.06 & 1.85 & 2.77 & 2.87 & 2.75 & 1.69 & $\mathbf{3 . 5 0}$ \\
FE & 115 & 120 & 144 & 180 & 65 & 130 & $\mathbf{1 3 5}(70 \mathrm{~F}, 65 \mathrm{E})$ & 0.35 & 1.43 & 1.53 & 1.77 & 1.45 & 3.37 & $\mathbf{3 . 6 0}$ \\
RU & 70 & 75 & 72 & 60 & 63 & 75 & $\mathbf{7 5}(35 \mathrm{R}, 40 \mathrm{U})$ & 0.35 & 1.43 & 1.63 & 1.77 & 1.45 & 2.11 & $\mathbf{2 . 3 0}$
\end{tabular}

would have made interfacing with the Maestro impossible, was eliminated (Fig. 3-e). Second, the RU capstan and transmission was relocated from the palmar side of the hand to the dorsal side (Fig. 3-f) so it would not interfere with the hand exoskeleton when grasping motions occur.

\section{Practical Considerations}

Several features have been introduced to make the device more functional for users, clinicians, and researchers alike. Addressing ergonomic downfalls of previous devices is a foam padded elbow support (Fig. 3-b) which can be adjusted laterally and vertically and fitted with small and large sized cuffs. The support preserves the integral assumption of exoskeletons by reducing user movement with respect to the exoskeleton, and avoids an oversight present in previous devices whereby subjects with fragile skin would come into contact with bare metal surfaces, pinch points, and fasteners.

Each joint integrates an in-line cable tensioning mechanism like the one shown in Fig. 4-b. With clinicians in mind, all joints can be quickly re-wrapped and tensioned when provided with a $1 / 4$ " wrench and pre-made cable sections. It is worth noting that the choice of cable was also upgraded to pre-stretched, flexible 7x19 strand core stainless steel which further reduces friction and prevents loosening with continued use.

Since ROM in the FE joint is asymmetrical, the ability to change between left-handed and right-handed configurations was implemented. Referencing Fig. 4-c, the RU module (left) would be detached from the FE module (right), itself detached from the PS module (center). Next, the FE actuator would be relocated to the left side of the PS module, the FE module flipped $180^{\circ}$ and reattached to the PS module, and the RU module moved to the right side of the FE module. Note that because the PS and RU modules' cable windings are self-contained, only the FE joint would require rewinding in the event of a configuration change.

Other improvements include: an upgrade from 6061-T6 to 7075-T6 aluminum alloys, allowing for reductions in thickness in multiple areas; the use of hybrid-ceramic ball bearings with $\mathrm{Si}_{3} \mathrm{~N}_{4}$ balls in the $\mathrm{FE}$ and RU joints, offering decreased friction and requiring no lubrication; and routing of electrical wires through joint axes to eliminate wire draping and drag (Fig. 4-d). Of particular interest is the application of a white polymer-ceramic coating. The coating provides a very low signature in infrared, making passive marker motion capture studies feasible, and has a high dielectric strength for compatibility with sEMG.
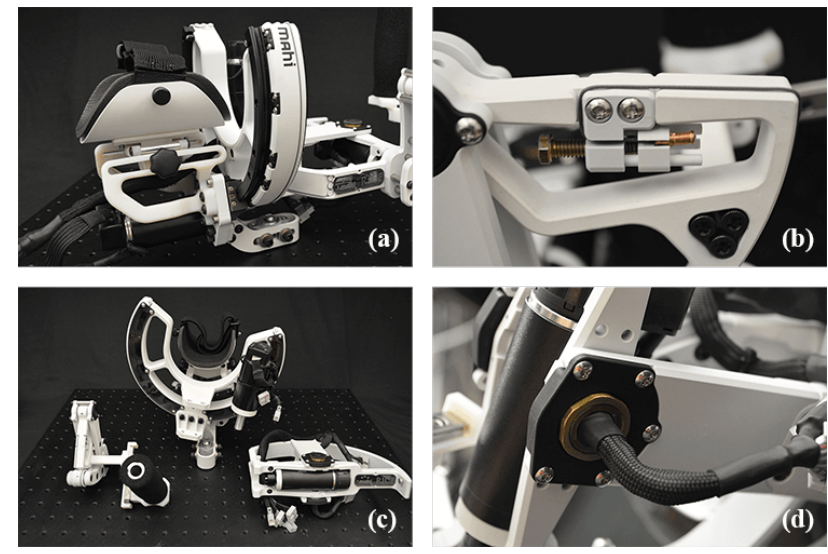

Fig. 4. OpenWrist Features - (a) central hub with curvilinear rails, (b) integrated quick connect tensioner, (c) modular assembly allows for ambidextrous configurations, (d) electrical wire routing through joint axes. 
- Vertical $\cdots \cdots 25^{\circ}$ Angled $---35^{\circ}$ Angled $--30^{\circ}$ Angled

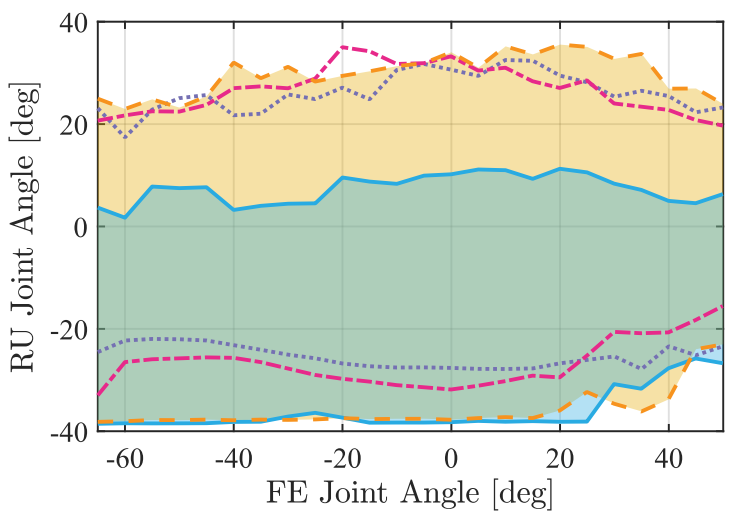

Fig. 5. ROM in the RU joint as a function of FE joint angle for the multiple grip styles evaluated. Shaded regions place emphasis on the workspace of the vertical grip and the final $30^{\circ}$ angled grip that was chosen.

\section{E. Hand Grip}

Although users are primarily intended to interface the OpenWrist via the Maestro hand exoskeleton, a hand grip was developed should wrist-only studies be conducted. Virtually all wrist exoskeletons, including those developed by our group, feature a grip that is vertically oriented when the exoskeleton is in its neutral position. An overlooked flaw with this style of grip is that it puts the wrist in an orientation that is already significantly radially deviated. Thus, the neutral orientations of the robot and user do not coincide. To address this, multiple grip angles (obtained by measuring the neutral grip angle of several individuals) were evaluated during the design phase by rastering the FE-RU workspace to within the user's comfort threshold. Fig. 5 maps the achievable ROM in the RU joint workspace as $\mathrm{FE}$ is varied in $5^{\circ}$ increments for four grips tested. Note the significant increase in the upper workspace limits from the vertical grip to the angled grips. However, simply introducing an angle, as with the $25^{\circ}$ and $35^{\circ}$ grips, also resulted in misalignment of joint axes and collision with the exoskeleton before reaching the lower workspace limits. The final grip (depicted in Fig. 2), has an altered geometry at its attachment point to regain this lost lower workspace, and is angled at $30^{\circ}$ based on user feedback. Compared with the traditional vertical grip, the new angled grip offers an increase of approximately $51 \%$ in FE-RU workspace area.

\section{F. Mechatronics and Controls}

All actuators are Maxon RE-series DC motors, each fitted with a Broadcom/Avago HEDL-5540 A11 optical encoder capable of 500 counts per revolution. Specific actuator details as well as transmission ratios and sensor resolutions at the joint are listed in Table II. Power is supplied from a Quanser VoltPAQ-X4 linear voltage-controlled amplifier running in a current control mode, and up to $4.16 \mathrm{~A}$ of continuous current can be provided to each actuator. The amplifier and encoders interface with MATLAB and Simulink through a Quanser Q8-USB data acquisition device and Quarc control software. The system is capable of operating at rates of up to $2 \mathrm{kHz}$ depending on the complexity of the controller.
TABLE II

ACTUATOR AND SENSOR DETAILS

\begin{tabular}{lccc} 
Joint & Actuator (PN) & Transmission & Sensor (Joint Resolution) \\
\hline PS & RE-40 (148877) & $1: 18.7$ & HEDL-5540 $\left(0.0096^{\circ}\right)$ \\
FE & RE-40 (148877) & $1: 19.2$ & HEDL-5540 $\left(0.0094^{\circ}\right)$ \\
RU & RE-30 (310009) & $1: 25.6$ & HEDL-5540 $\left(0.0070^{\circ}\right)$
\end{tabular}

\section{CHARACTERIZATION}

In this section, we present the experimental characterization of the OpenWrist including position bandwidth, static and kinetic friction, viscous damping coefficients, and inertial elements. Each of the experiments discussed was performed on all three joints. To isolate nonrigid body effects, gravitational disturbances were eliminated by orienting the device such that the axis of the joint in question was parallel with the direction of gravity. The remaining two joints were locked with a high proportional gain PD controller, and the passive DOF on the grip was secured. For consistency, the specific characterization experiments conducted match those used for our group's other devices [7], [8] with the exception of the bandwidth test which previously utilized a chirp signal input.

\section{A. Inertia, Viscous Damping, and Kinetic Friction}

The dynamic properties of the device were investigated by adopting the model and logarithmic decrement techniques described in [21]. By examining the step response of the underdamped system, the inertial, viscous, and dry friction contributions to exponential decay can be isolated.

Since the physical system displays effectively zero stiffness, a proportional controller was implemented with the

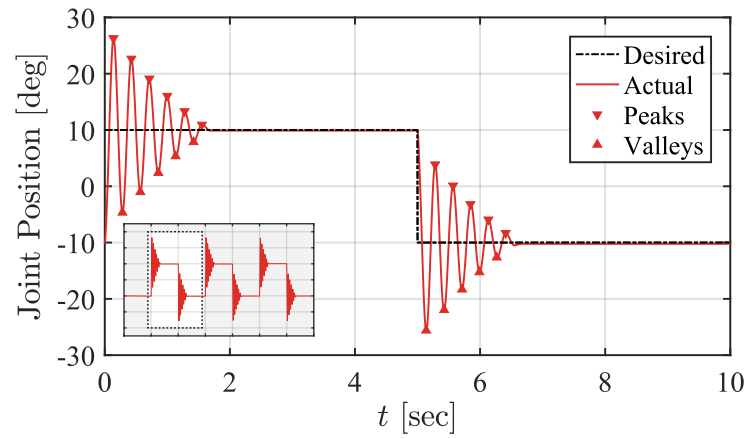

Fig. 6. One of three step response cycles about $0^{\circ}$ for the PS joint.

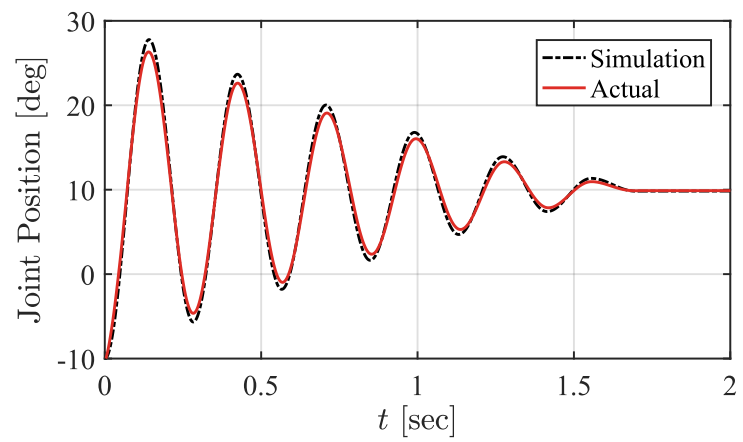

Fig. 7. Top response from Fig. 6 when overlaid with the simulated response 


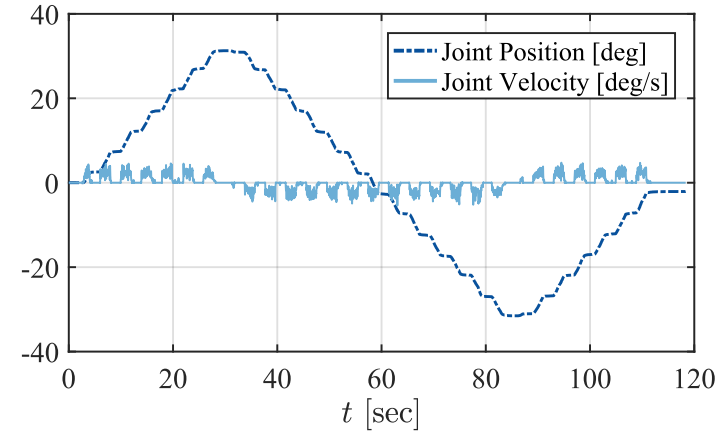

Fig. 8. Position and velocity of the RU joint during the static friction ramp test.

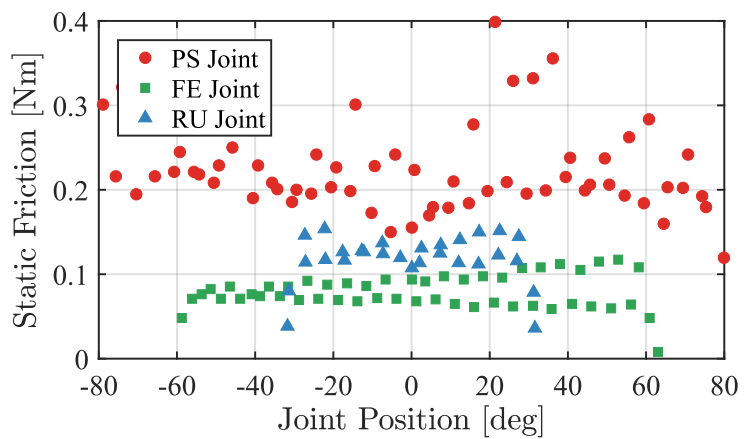

Fig. 9. Static friction of the PS, FE, and RU joints taken during the ramp test and plotted along their respective workspaces.

actuator set to behave as a relatively soft spring with spring constants of 15,5 , and $8 \mathrm{Nm} / \mathrm{rad}$ for PS, FE, and RU, respectively. A square wave position input with a step-to-step amplitude of $20^{\circ}$ was commanded, and 3 complete cycles were recorded. To cover most of the joint workspace, the test was conducted about starting joint angles of $-50^{\circ}, 0^{\circ}$, $50^{\circ}$ for PS; $-30^{\circ}, 0^{\circ}, 30^{\circ}$ for $\mathrm{FE}$; and $-5^{\circ}, 0^{\circ}, 5^{\circ}$ for RU. Peaks and valleys were extracted from the underdamped response separately for both the top and bottom responses (Fig. 6). From each response, the joint's inertia, viscous damping coefficient, and kinetic friction parameters were calculated. The average values across all responses and starting angles are given in Table III. To validate the accuracy of the model, the averaged parameters and proportional gain constant were used to simulate the model presented in [21]. A representative simulated response is shown in Fig. 7.

\section{B. Static Friction}

To investigate static friction, multiple position ramps were commanded across the workspace of each joint. The input ramps up or down $5^{\circ}$ over 2 seconds, pauses for an additional 2 seconds, and then continues ramping in this manner until the extreme points of the workspace have been reached (Fig. 8). Static friction is inferred from the commanded torque when movement is initiated, i.e., one time step before the instant the backwards-differentiated velocity becomes nonzero near the beginning of each ramp. Therefore, detecting subtle changes in velocity were more important than accurate position control, so a soft proportional controller was used. Static friction as a function of joint workspace is shown in Fig. 9, with average and max values highlighted in Table III.

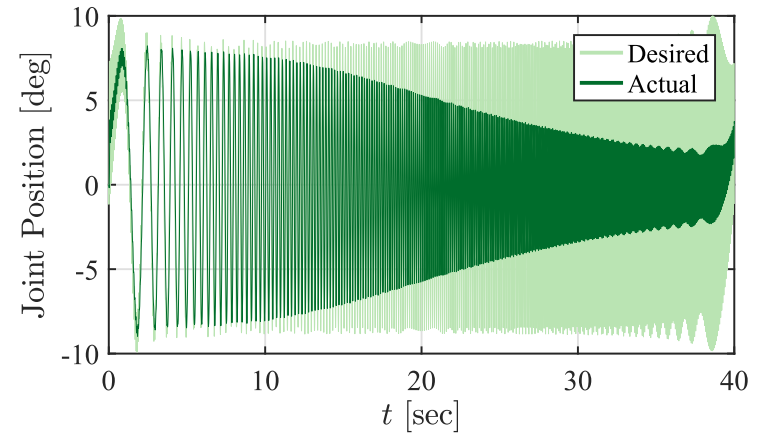

Fig. 10. A Schroeder multisine input excites the FE joint through a range of increasing frequencies while remaining constant in the power spectrum.

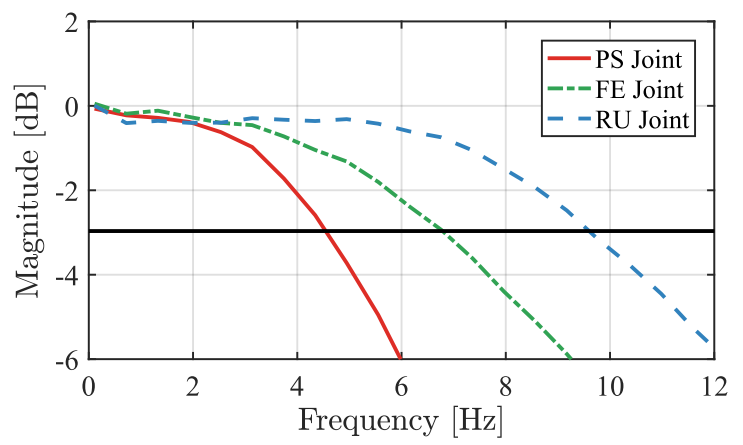

Fig. 11. Bode plots obtained by estimating the transfer function of Fig 10 . Bandwidth values, defined by the $-3 \mathrm{~dB}$ cutoff, are $4.7,7.0$, and $9.8 \mathrm{~Hz}$ for the PS, FE, and RU joints, respectively.

\section{Closed-Loop Position Bandwidth}

Since the device may employ a position control strategy in the future, it is important to determine the closed-loop position bandwidth. A critically damped PD controller was implemented, and a Schroeder multisined excitation signal conditioned between $-10^{\circ}$ and $10^{\circ}$ was used as the position input. Fig. 10 shows a representative plot of the commanded versus actual positions, with attenuation beginning around the 10 second mark. Fig. 11 provides the Bode plot for each DOF with the bandwidth cutoff of $3 \mathrm{~dB}$ clearly shown. The bandwidth values are provided in Table III.

\section{DISCUSSION}

Characterization of the OpenWrist underscores the significance of the numerous design considerations likely to improve its potential as a rehabilitative device. The model from [21] captures the dynamic properties with reasonable accuracy despite its simplicity (Fig. 7). Compared to the RiceWrist-S, inertia reductions of $12 \%$ and $21 \%$ are achieved in the FE and RU joints, respectively, as a result of lower weight components and strategically placed actuators. Hybrid-ceramic ball bearings and improved capstan-cable windings contribute to decreases in maximum static friction by $47 \%$ in FE and $27 \%$ in RU. The separation of FE static friction measurements shown between $40^{\circ}$ and $60^{\circ}$ in Fig. 9 suggests that the test was affected by gravity. The effect remained repeatable despite multiple attempts to eliminate it and is likely an outcome of the FE module's asymmetric design. The inconsistent static friction at the extremes of the RU workspace are explained by a build-up and release of 
TABLE III

Average Device Characteristics

\begin{tabular}{lcccccccccc} 
& \multicolumn{2}{c}{ Inertia $\left[\mathrm{kg} \cdot \mathrm{m}^{2}\right]$} & \multicolumn{2}{c}{ Viscous Coeff. $\left[\frac{\mathrm{Nm} \cdot \mathrm{s}}{\mathrm{rad}}\right]$} & \multicolumn{2}{c}{ Kinetic Friction $[\mathrm{Nm}]$} & \multicolumn{2}{c}{ Static Friction $(\mathrm{Max})[\mathrm{Nm}]$} & \multicolumn{2}{c}{ Bandwidth $[\mathrm{Hz}]$} \\
Joint & RW-S & OpenWrist & RW-S & OpenWrist & RW-S & OpenWrist & RW-S & OpenWrist & RW-S & OpenWrist \\
\hline PS & 0.0258 & 0.0305 & 0.428 & 0.0252 & n/a & 0.1891 & n/a $(0.221)$ & $0.2250(0.3990)$ & 3.5 & 4.6 \\
FE & 0.0134 & 0.0119 & 0.085 & 0.0019 & n/a & 0.0541 & n/a $(0.198)$ & $0.0720(0.1042)$ & 6.0 & 7.0 \\
RU & 0.0048 & 0.0038 & 0.135 & 0.0029 & n/a & 0.1339 & n/a $(0.211)$ & $0.1180(0.1537)$ & 8.3 & 9.8
\end{tabular}

cable tension during directional changes near the edges.

Although the curvilinear rails resulted in increased inertia and static friction in the PS joint, the open design is of far greater importance. Note the periodic spikes in PS static friction shown in Fig. 9; these spikes roughly correlate with the gaps between the four rail segments. Thus, the authors suspect that the high static friction value is likely due to a slight misalignment of the rails. This issue can be expected to improve with continued adjustment and breakin. Furthermore, because torque output on the PS joint has been doubled, any undesired effects of increased inertia and friction can be compensated for in control implementation.

Kinetic friction values measured for the OpenWrist consume a maximum of only $6 \%$ of the continuous torque output in any joint. Closed-loop position bandwidth is increased over the RiceWrist-S across the board and either exceeds or is slightly less than the $5 \mathrm{~Hz}$ achievable by humans in uncontrolled motions.

While not discussed in this paper, the OpenWrist was further characterized and validated in two separate subject studies involving wrist pointing tasks. The effects of the OpenWrist's dynamic properties on movement smoothness during wrist pointing tasks are characterized in [22], while wrist pointing trajectories as recorded by robot encoders and passive marker motion capture are compared in [23].

\section{CONCLUSION}

The READAPT wrist module, the OpenWrist, meets the design goals for coordinated hand-wrist exoskeletons previously outlined. Compatibility with the Maestro handexoskeleton is insured by eliminating obtrusive geometry present in the previously used RiceWrist-S, and relocating the RU module so that grasping motions can occur. The introduction of an open PS design makes donning and doffing for impaired users feasible and further allows for the Maestro to be donned beforehand. The device exceeds the requirements of ADL for both torque and ROM in all joints. Ergonomics are also addressed with the addition of an adjustable foam padded elbow support and $30^{\circ}$ angled grip for standalone mode. Additional practical improvements allowing for rapid maintenance and ambidextrous reconfiguration enhance its effectiveness in a clinical setting.

\section{REFERENCES}

[1] P. S. Lum et al., "Robotic approaches for rehabilitation of hand function after stroke," American J. of Physical Medicine \& Rehab., vol. 91, no. 11, pp. S242-S254, 2012.

[2] D. Mozaffarian et al., "Heart disease and stroke statistics-2016 update," Circulation, 2015.
[3] C. Bütefisch et al., "Repetitive training of isolated movements improves the outcome of motor rehabilitation of the centrally paretic hand," J. of the Neuro. Sciences, vol. 130, no. 1, pp. 59-68, 1995.

[4] C. N. Schabowsky et al., "Development and pilot testing of HEXORR: Hand EXOskeleton Rehabilitation Robot," J. of Neuroengineering and Rehab., vol. 7, no. 36, pp. 1-16, 2010.

[5] M. Bouzit et al., "The Rutgers Master II-new design force-feedback glove," IEEE/ASME Trans. on Mechatronics, vol. 7, no. 2, pp. 256263, 2002.

[6] M. Cempini et al., "A Powered Finger-Thumb Wearable Hand Exoskeleton With Self-Aligning Joint Axes," IEEE/ASME Trans. on Mechatronics, vol. 20, no. 2, pp. 705-716, 2015.

[7] A. U. Pehlivan et al., "Design and validation of the RiceWrist-S exoskeleton for robotic rehabilitation after incomplete spinal cord injury," Robotica, vol. 32, no. 8, pp. 1415-1431, 122014.

[8] J. A. French et al., "System characterization of MAHI Exo-II: a robotic exoskeleton for upper extremity rehabiliation," in ASME Dynamic Systems and Controls Conf., vol. 3. San Antonio, TX: ASME, 2014.

[9] H. I. Krebs et al., "Robot-aided neurorehabilitation: A robot for wrist rehabilitation," IEEE Trans. on Neural Systems and Rehab. Engineering, vol. 15, no. 3, pp. 327-335, Sept 2007.

[10] J. A. Martinez et al., "Design of wrist gimbal: A forearm and wrist exoskeleton for stroke rehabilitation," in IEEE Intl. Conf. on Rehab. Robotics (ICORR), June 2013, pp. 1-6.

[11] L. Cappello et al., "Evaluation of wrist joint proprioception by means of a robotic device," in 2014 11th Intl. Conf. on Ubiquitous Robots and Ambient Intelligence (URAI), Nov 2014, pp. 531-534.

[12] S. Ates et al., "Combined active wrist and hand orthosis for home use: Lessons learned," in IEEE Intl. Conf. on Rehab. Robotics (ICORR), Aug 2015, pp. 398-403.

[13] Z.-M. Li, "The influence of wrist position on individual finger forces during forceful grip," The J. of Hand Surgery, vol. 27, no. 5, pp. 886-896, 2002.

[14] A. D. Deshpande et al., "Contributions of intrinsic visco-elastic torques during planar index finger and wrist movements," IEEE Trans. on Biomedical Eng., vol. 59, no. 2, pp. 586-594, 2012.

[15] P.-H. Kuo and A. D. Deshpande, "Contribution of passive properties of muscle-tendon units to the metacarpophalangeal joint torque of the index finger," in IEEE RAS and EMBS Intl. Conf. on Biomedical Robotics and Biomechatronics (BioRob), 2010, pp. 288-294.

[16] J. S. Knutson et al., "Intrinsic and extrinsic contributions to the passive moment at the metacarpophalangeal joint," J. of Biomechanics, vol. 33, no. 12 , pp. $1675-1681,2000$.

[17] C. G. Rose et al., "Characterization of a hand-wrist exoskeleton, READAPT, via kinematic analysis of redundant pointing tasks," in IEEE Intl. Conf. on Rehab. Robotics (ICORR), Aug 2015, pp. 205210.

[18] P. Agarwal et al., "Design, control and testing of a thumb exoskeleton with series elastic actuation," Intl. J. of Robotics Research, 2017.

[19] K. D. Fitle et al., "A robotic exoskeleton for rehabilitation and assessment of the upper limb following incomplete spinal cord injury," in IEEE Intl. Conf. on Robotics and Automation (ICRA). IEEE, 2015, pp. 4960-4966.

[20] A. U. Pehlivan et al., "Mechanical design of a distal arm exoskeleton for stroke and spinal cord injury rehabilitation," in IEEE Intl. Conf. on Rehab. Robotics (ICORR), June 2011, pp. 1-5.

[21] J. W. Liang and B. F. Feeny, "Identifying coulomb and viscous friction from free-vibration decrements," Nonlinear Dynamics, vol. 16, no. 4, pp. 337-347, 1998.

[22] A. Erwin et al., "The effect of robot dynamics on smoothness during wrist pointing," in IEEE Intl. Conf. on Rehab. Robotics (ICORR), 2017.

[23] C. G. Rose et al., "Estimating anatomical wrist joint motion with a robotic exoskeleton," in IEEE Intl. Conf. on Rehab. Robotics (ICORR), 2017. 